Copyright(C)2012. The Korean Academy of Tuberculosis and Respiratory Diseases. All rights reserved.

\title{
Sarcoidosis Presenting with Massive Pleural Effusion and Elevated Serum and Pleural Fluid Carbohydrate Antigen-125 Levels
}

\author{
In Seon Lee, M.D., Sae Byul Kim, M.D., Chan Soo Moon, M.D., Sung Mo Jung, M.D., Song Yee Kim, M.D., \\ Eun Young Kim, M.D., Ji Ye Jung, M.D., Young Ae Kang, M.D., Ph.D., Young Sam Kim, M.D., Ph.D., Se \\ Kyu Kim, M.D., Ph.D., Joon Chang, M.D., Ph.D., Moo Suk Park, M.D., Ph.D. \\ Division of Pulmonary and Critical Care Medicine, Department of Internal Medicine, The Institute of Chest Diseases, Yonsei \\ University College of Medicine, Seoul, Korea
}

\begin{abstract}
A 55-year-old woman was admitted for an elevated serum carbohydrate antigen-125 (CA-125) level, and a left pleural effusion, which were detected at a routine health examination. Computed tomography of the chest was performed upon admission, revealing extensive bilateral paratracheal and mediastinal lymph node enlargement with a massive left-sided pleural effusion. Subsequent analysis of the pleural fluid demonstrated consistency with an exudate, no evidence of malignant cells, and a normal adenosine deaminase. However, the pleural fluid and serum CA-125 levels were 2,846.8 U/mL and $229.5 \mathrm{U} / \mathrm{mL}$, respectively. A positron emission tomography did not reveal any primary focus of malignancy. Finally, a surgical mediastinoscopic biopsy of several mediastinal lymph nodes was performed, revealing non-necrotizing granulomas, consistent with sarcoidosis. After a month of treatment of prednisolone, the left pleural effusion had resolved, and after 2 months the serum CA-125 level was normalized.
\end{abstract}

Key Words: Sarcoidosis; Pleural Effusion; CA-125 Antigen

\section{Introduction}

Massive pleural effusions can be produced by many causes, including sarcoidosis. However, pleural involvement of sarcoidosis is rare, with an incidence in the literature of approximately $1 \sim 10 \%{ }^{1}$. Carbohydrate (or cancer) antigen-125 (CA-125) is a glycoprotein that has been associated with ovarian carcinoma and several other non-gynecologic malignancies ${ }^{2}$. Herein, we report a case of a massive pleural effusion due to pleural sarcoidosis, with associated increases in pleural and serum

Address for correspondence: Moo Suk Park, M.D., Ph.D. Division of Pulmonary and Critical Care Medicine, Department of Internal Medicine, Yonsei University College of Medicine, 50, Yonsei-ro, Seodaemun-gu, Seoul 120-752, Korea

Phone: 82-2-2228-1930, Fax: 82-2-393-6884

E-mail: pms70@yuhs.ac

Received: Apr. 5, 2012

Revised: Apr. 24, 2012

Accepted: Jul. 5, 2012

(c) It is identical to the Creative Commons Attribution Non-Commercial License (http://creativecommons. org/licenses/by-nc/3.0/).
CA-125 levels that normalized after steroid therapy.

\section{Case Report}

A 55-year-old woman was admitted for further evaluation of an elevated serum CA-125 and a left pleural effusion identified by her primary care physician at a routine health examination. At the time, the patient complained of a mild dry cough and dyspnea on exertion which had been present for several months. She also endorsed an unintentional weight loss of $3 \mathrm{~kg}$ during the preceding 2 months.

The patient had a history of a hysterectomy with bilateral salpingo-oophorectomy for treatment of uterine myomatosis 5 years previously. She has been taking medicine for hypertension, and denied any history of tuberculosis. She had no specific contributory family history. She had no history of tobacco or alcohol use. She was a housewife. 


\section{Physical examinations}

The blood pressure was 128/83 $\mathrm{mm} \mathrm{Hg}$, the pulse rate was 80 beats per minute, the body temperature was $36.1^{\circ} \mathrm{C}$, the respiratory rate was 18 breaths per minute, and the oxygen saturation was 100\% while breathing ambient air. There was no palpable lymph node enlargement in the head, neck, or axillae. The lung sounds were noted to be dull to percussion at the left hemithorax, and diminished at the left base. The remainder of the physical examination was normal.

\section{Laboratory findings}

The serum CA-125 was significantly elevated (229.5 $\mathrm{U} / \mathrm{mL}$ ), as was the serum angiotensin-converting enzyme (119 U/L). All other tumor markers were within normal limits. Other test results were also normal, including white blood cell and platelet counts, measurements of electrolytes, glucose, protein, albumin, globulin, and bilirubin, tests of hepatic and renal function, and urinalysis. An acid-fast bacillus (AFB) smear of the patient's sputum was negative, with no organisms observed on sputum culture.

Thoracentesis and pleural biopsy were then performed, yielding $500 \mathrm{~mL}$ of clear yellow pleural fluid.
The pleural fluid analysis revealed an exudative pattern, with a protein level of $5.6 \mathrm{~g} / \mathrm{dL}$ (the serum protein level, $7.3 \mathrm{~g} / \mathrm{dL}$ ), a lactate dehydrogenase level of $269 \mathrm{IU} / \mathrm{L}$ (the serum lactate dehydrogenase level, 322 IU/L), a glucose of $39 \mathrm{mg} / \mathrm{dL}$, an albumin of $3.4 \mathrm{~g} / \mathrm{dL}$ and $\mathrm{pH}$ of 7.2 . Additionally, a cell count of the pleural fluid was performed showing 4,850 cells/ $\mu \mathrm{L}$ (polymorphonuclear cells 0\%, lymphocytes 100\%, eosinophils 0\%, basophils $0 \%$, and other cells 0\%), with a pleural fluid CA-125 concentration of 2,846.8 U/mL and an adenosine deaminase concentration of $37.8 \mathrm{IU} / \mathrm{L}$. No malignant cells were observed on cytology. All bacterial, fungal and mycobacterial cultures of the pleural fluid were negative. The results of pulmonary function tests were within normal limit, and an electrocardiogram was normal.

\section{Image findings}

A chest radiograph showed findings consistent with a massive left-sided pleural effusion and bilateral hilar prominence (Figure 1A). A contrast-enhanced computed tomography (CT) of the chest demonstrated extensive bilateral paratracheal and mediastinal lymphadenopathy, and left-sided pleural effusion (Figure 2A), without any lesions involving the lung parenchyma. A

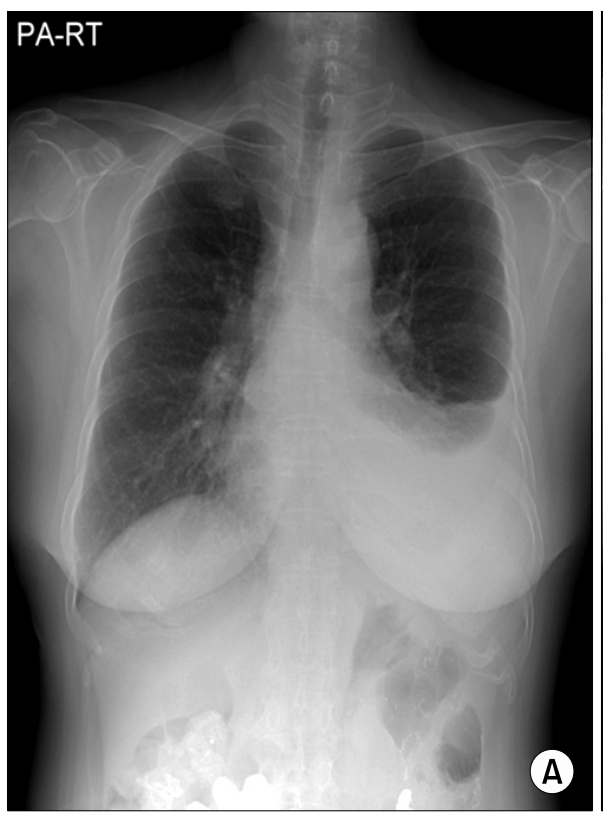

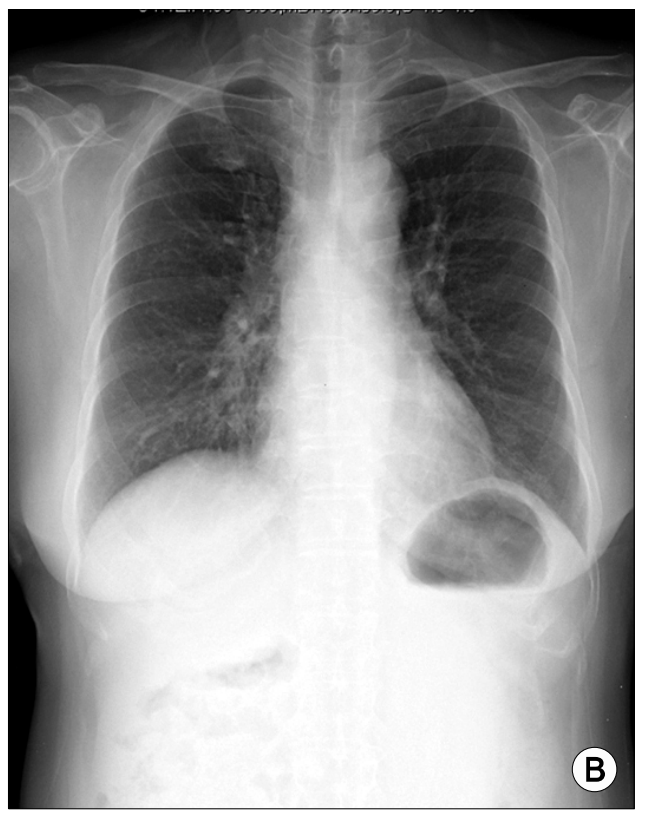

Figure 1. (A) The initial chest $X$-ray showed a leftsided massive pleural effusion, which disappeared after 2 months of medication with oral prednisolone (B). 


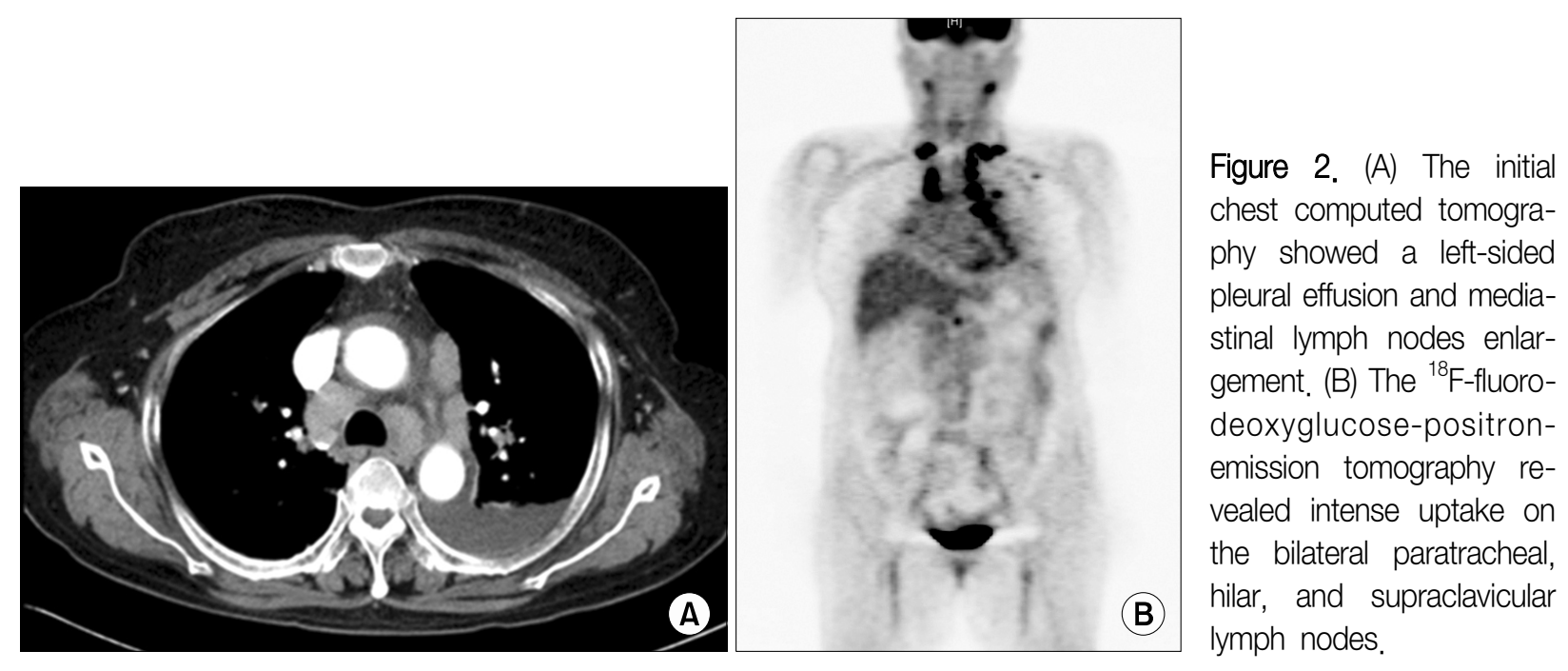

subsequent ${ }^{18}$ F-fluorodeoxyglucose-positron-emission tomography $\left({ }^{18}\right.$ F-FDG-PET) revealed FDG activity in the bilateral paratracheal, hilar, and supraclavicular lymph nodes, which were suspicious for malignancy. However, there was no evidence of any other specific focus of malignancy (Figure 2B). Similarly, the patient's breast ultrasonography, mammography, and Pap smear at the most recent health care examination did not indicate any malignant focus, nor did the esophageal gastroduodenoscopy or colonoscopy. A contrast-enhanced CT of the abdomen likewise revealed no significant abnormal findings.

\section{Bronchoscopic findings}

On bronchoscopic examination, there was mild blunting at the main carina, and there were multiple elevated nodules in the proximal medial walls of the left second carina and the left lower lobar bronchus. There was no evidence of respiratory viruses, fungi, or bacteria. And the result of AFB smear in the bronchial washing specimen was negative.

\section{Histopathological findings}

Pleural fluid cytology was negative for malignant cells, whereas the pleural biopsy showed chronic granulomatous inflammation with giant cells. Ziehl-Neelsen and diastase-pretreated periodic acid-Schiff stains revealed no infectious organisms. Pathologic findings from multiple nodules of the fiberoptic bronchoscopic biopsy revealed non-necrotizing granulomas in the bronchial wall, suggesting no definitive findings (Figure $3 \mathrm{~A}$ ), and transbronchial needle aspiration cytology was negative for malignancy. Finally, a surgical mediastinoscopic biopsy of the right paratracheal lymph nodes was performed, showing non-necrotizing granulomas consistent with sarcoidosis (Figure 3B). Taken together, pleural sarcoidosis with lymph node and endobronchial involvement was diagnosed.

\section{Treatment and progress}

The patient was started on a treatment regimen of 1 $\mathrm{mg} / \mathrm{kg}$ oral prednisolone. After one month, the left pleural effusion was resolved (Figure 1B), and the serum angiotensin converting enzyme level was decreased from $119 \mathrm{U} / \mathrm{L}$ to $18 \mathrm{U} / \mathrm{L}$. After 2 months of oral prednisolone therapy, the serum CA-125 level was normalized (229 U/mL to $34 \mathrm{U} / \mathrm{mL}$ ). No signs of relapse were observed during a 5-month steroid tapering period, and the pleural effusion never recurred during the subsequent 5-year follow-up period after discontinuing the steroids.

\section{Discussion}

Sarcoidosis is a multisystem disease of unknown origin, resulting in classic granulomatous-type inflamma- 

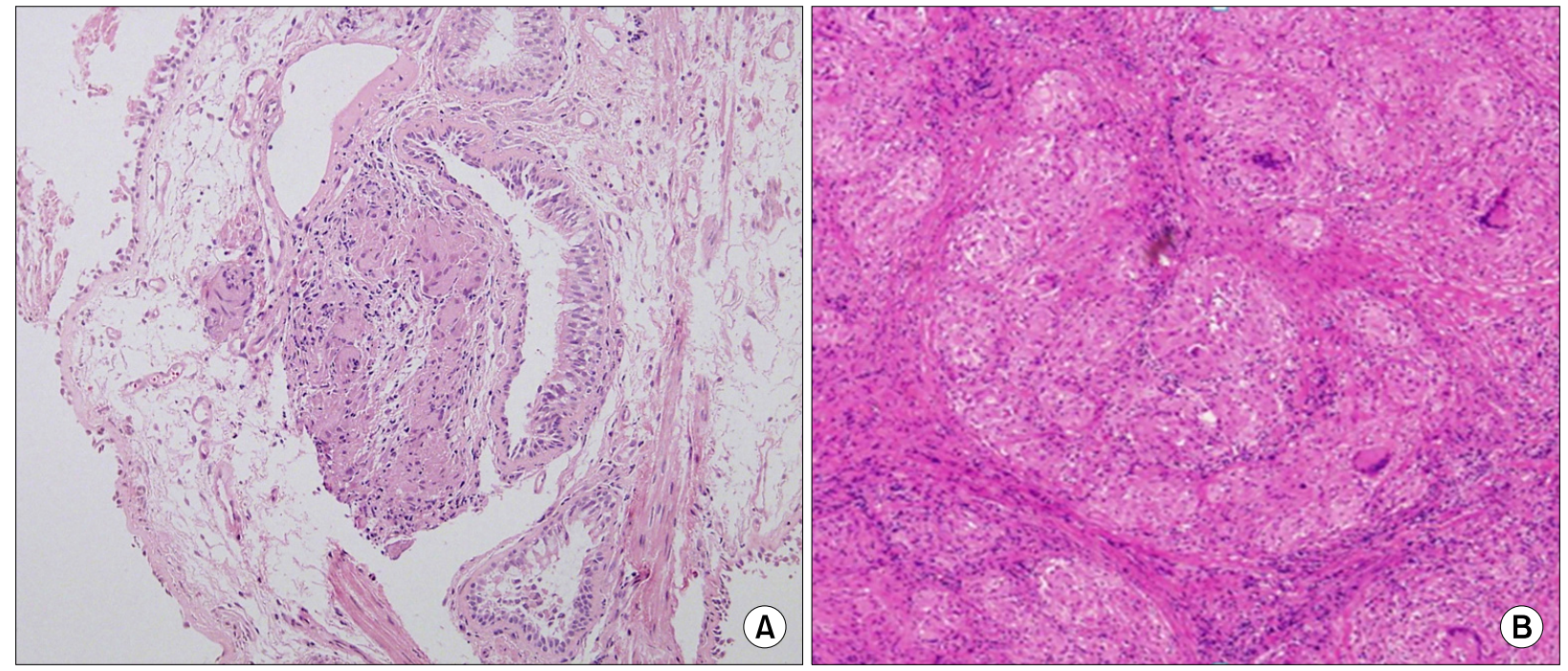

Figure 3. (A) The fiberoptic bronchoscopic biopsy revealed non-necrotizing granulomas in the bronchial wall (H\&E stain, $\times 100$ ). (B) The mediastinoscopic biopsy of right paratracheal lymph node revealed that all normal structures were entirely replaced by non-caseating granulomas, each composed of aggregates of tightly clustered epithelioid cells (H\&E stain, $\times 400)$.

tion $^{3}$. Sarcoidosis involving the pleura is rare, and usually manifests itself as non-specific pleural thickening, pleural effusion, and pneumothorax. Huggins et al. ${ }^{1}$ reported that only 2 of 181 sarcoidosis patients (1.1\%) was observed a pleural effusion secondary to sarcoidosis via chest ultrasonography. Moreover, patients with pleural sarcoidosis usually have pulmonary involvement.

Pleural effusion secondary to sarcoidosis can present unilaterally or bilaterally, with the right side (45\%) more often affected than the left $(33 \%)^{4}$ for unclear reasons. In most cases, the actual amount of pleural effusion is small to moderate, though some cases of massive pleural effusion have been reported. Furthermore, the pleural effusion type associated with sarcoidosis has generally been a lymphocyte-predominant exudate, though a transudate with a predominance of eosinophils has occurred in a minority of cases 5 .

The diagnosis of sarcoidosis is supported by clinical and radiologic findings, in addition to histopathology consistent with noncaseating granuloma in one or more organs. Notably, no pathognomonic histologic features exist for granulomas associated with sarcoidosis. Moreover, a pleural biopsy sample demonstrating non- caseating granulomas is required to diagnose pleural sarcoidosis, and all other causes of pleural effusion must be ruled out, such as tuberculosis, malignancy, or congestive heart failure ${ }^{6}$. Specifically, tuberculous granulomas show caseation, which can help distinguish tuberculoma from sarcoidosis, though this feature may not be present in all the cases. As such, all biopsy specimens should be thoroughly evaluated for underlying infectious agents, particularly Mycobacterium tuberculosis.

CA-125 is a high molecular glycoprotein originating from embryonic coelomic epithelia, of which the fallopian tubes, endometrium, endocervix, pleura, pericardium, and peritoneum are derived ${ }^{2}$. Serum CA-125 levels are often used as a tumor marker in ovarian carcino$\mathrm{ma}$, as it is often initially elevated and decreases after chemotherapy or tumor resection. However, serum CA-125 levels may also be elevated in the setting of non-gynecological malignancies (including lung cancer and non-Hodgkin's lymphoma), and various other benign conditions (such as liver disease, ascites, connective tissue disease, and chronic constrictive pericarditis). Furthermore, some evidence suggests that granulomatous conditions can also cause elevations in serum CA-125. For instance, tuberculosis can result in 
massive pleural effusions with secondary elevation of CA-125 . Additionally, CA-125 elevation has been associated with cases of peritoneal, pleural, and pulmonary sarcoidosis $^{2}$.

In our patient, underlying liver, renal, or rheumatologic disease were not ever identified, and there were not any evidence to suggest past or present tuberculous infection. To exclude malignancy and other associated benign conditions, an extensive workup was performed, including ${ }^{18}$ F-FDG-PET, contrast-enhanced CT of the abdomen, esophageal gastroduodenoscopy, colonoscopy, mammography, and breast ultrasonography, which identified no other cause that could underlie the elevated serum CA-125. In this way, sarcoidosis was felt to be the only etiologic factor resulting in the elevated serum CA-125 and large pleural effusion, without pulmonary nor peritoneal involvement ${ }^{2}$.

The treatment of sarcoidosis with pleural involvement should be determined on a case-by-case basis, as these effusions usually resolve spontaneously within 1 $\sim 3$ months ${ }^{4,6}$. Systemic corticosteroids should only be considered in patients with symptomatic disease and for cases of recurrent pleural effusion, as persistent pleural effusions may lead to chronic pleural irritation, pleural thickening, and ultimately fibrothorax. Our patient suffered from progressive dyspnea on exertion with an increasingly massive pleural effusion, and was thus suc- cessfully treated with systemic corticosteroids.

In conclusion, sarcoidosis can be the cause of massive pleural effusion as well as elevated serum CA-125 levels. Clinicians should consider sarcoidosis as a differential diagnosis in patients with massive pleural effusion or elevated serum CA-125, even without pulmonary or peritoneal involvement.

\section{References}

1. Huggins JT, Doelken P, Sahn SA, King L, Judson MA. Pleural effusions in a series of 181 outpatients with sarcoidosis. Chest 2006;129:1599-604.

2. Kalluri M, Judson MA. Sarcoidosis associated with an elevated serum CA 125 level: description of a case and a review of the literature. Am J Med Sci 2007;334:441-3.

3. Salerno D. Sarcoidosis pleural effusion: a not so common feature of a well known pulmonary disease. Respir Care 2010;55:478-80.

4. Soskel NT, Sharma OP. Pleural involvement in sarcoidosis. Curr Opin Pulm Med 2000;6:455-68.

5. Durand DV, Dellinger A, Guerin C, Guerin JC, Levrat R. Pleural sarcoidosis: one case presenting with an eosinophilic effusion. Thorax 1984;39:468-9.

6. Cohen M, Sahn SA. Resolution of pleural effusions. Chest 2001;119:1547-62.

7. Mansour M, Linden ER, Colby S, Posner G, Marsh F Jr. Elevation of carcinoembryonic antigen and CA-125 in a patient with multivisceral tuberculosis. J Natl Med Assoc 1997;89:142-3. 